\title{
Altered interferon- $\gamma$ expression in lymphocytes as a potential peripheral marker of chronic stress-induced cognitive deficit
}

\author{
María Laura Palumbo ${ }^{\mathrm{b}, *}$, María Emilia Di Rosso ${ }^{\mathrm{a}, \mathrm{c}}$, Elias Hugo Simon ${ }^{\mathrm{a}}$, \\ María Rosa Gonzalez Murano ${ }^{c}$, Ana María Genaro ${ }^{\mathrm{a}, \mathrm{c}, *}$ \\ ${ }^{a}$ Universidad de Buenos Aires (UBA), Facultad de Medicina, Primera Cátedra de Farmacología, Paraguay 2155, Piso 15, 1121 C.A.B.A, Argentina \\ b Centro de Investigaciones y Transferencia del Noroeste de la Provincia de Buenos Aires (CIT NOBA) - Universidad Nacional del Noroeste de la Provincia de Buenos Aires \\ (UNNOBA) - Consejo Nacional de Investigaciones Científicas y Técnicas (CONICET), Monteagudo 2772, 2700 Pergamino, Buenos Aires, Argentina \\ ${ }^{\mathrm{c}}$ Instituto de Investigaciones Biomédicas (BIOMED) - Pontificia Universidad Católica Argentina (UCA) - Consejo Nacional de Investigaciones Científicas y Técnicas \\ (CONICET), Av. Alicia Moreau de Justo 1600, Piso 3, 1107 C.A.B.A, Argentina
}

A R T I C L E I N F O

\section{Keywords:}

Chronic stress

Cytokines

Hippocampus

Lymph nodes

Glatiramer acetate

\begin{abstract}
A B S T R A C T
It is known that long-term exposure to stressful situations can produce severe consequences affecting behavioral, endocrine and immunological parameters. We have previously shown that stressed BALB/c mice had poor learning performance, which was reverted by glatiramer acetate treatment through a mechanism that likely involved the regulation of the cytokine balance and adult neurogenesis. In addition, recent results suggest that cytokine and neurotrophin expression in the hippocampus displayed similar tendencies as those in the serum. However, if lymphoid cells could be good candidates as peripheral markers of memory impairment have not yet been investigated. For this purpose, we analyzed the spatial memory and the neutrophin and cytokine mRNA levels in lymph nodes and hippocampus in mice submitted to chronic stress treated or not with glatiramer acetate. Results indicated that there was a correlation between the cytokine and neurotrophin mRNA levels in the hippocampus and in the peripheral lymph nodes, and the cognitive performance in BALB/c mice. In particular, our results suggest that altered IFN- $\gamma$ levels could be used as peripheral biomarker of cognitive deficit and treatment response.
\end{abstract}

\section{Introduction}

Nowadays the stress affects thousands of people around the world. Stress is a necessary survival mechanism of an organism in order to give an adequate response to external and/or internal stimuli. When these disturbing factors are prolonged in time, deleterious effects affecting the nervous, endocrine and immunological systems can be seen [1]. Among these parameters, the hippocampus, limbic area involved in learning and memory, is particularly vulnerable to the effects of stress [2]. Thus, it has been well documented in different animal models that chronic stress reduces adult neurogenesis in the dentate gyrus of the hippocampus $[2,3]$.

The central nervous system and the immune system are known to be engaged in an intensive bidirectional crosstalk which can be affected by stress and which involves multiple mediators, including cytokines and neurotrophins [4]. Neurotrophins are factors that induce the survival, development and function of neurons and have been involved in the pathophysiology of suicide [5] and depression [6]. The neurotrophin family includes nerve growth factor (NGF), brain-derived neurotrophic factor (BDNF) and neurotrophin-3 (NT-3). Cytokines are generally secreted by cells of the immune system, however they may also be synthesized and secreted by non-immune cells in order to signal immune cells. The balance between Th1 and Th2 responses is needed for optimal health whereas any disturbance may lead to pathological processes such as autoimmune diseases, sleep disturbance, major depression and other disorders $[7,8]$.

In previous reports, we have found in stressed female BALB/c mice cognitive decline evaluated in several behavioral test as open field, Ymaze, passive avoidance and object-in-place-task [9-11]. These behavioral alterations were correlated to a decrease in adult neurogenesis [3] and decrease in Th1/Th2 balance [3,10].

Glatiramer acetate (GA), which consists of acetate salts with

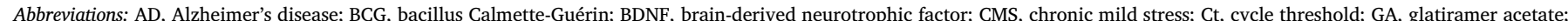
GAPDH, glyceraldehyde 3-phosphate dehydrogenase; GLM, General Linear Model; LSD, Least Significant Difference; NGF, nerve growth factor; NT-3, neurotrophin-3; Th, T helper

* Corresponding authors at: UBA-Fac. de Medicina-Primera Cát. de Farmacología, Paraguay 2155, floor 15, (1121), C.A.B.A, Argentina (A.M. Genaro). CIT NOBA-UNNOBA-CONICET, Jorge Newbery 261, (B6000), Junín, Buenos Aires, Argentina (M.L. Palumbo)

E-mail addresses: molecula_21@yahoo.com.ar (M.L. Palumbo), amgenaro@yahoo.com.ar (A.M. Genaro). 
synthetic polypeptides containing 1-alanine, l-glutamate, 1-lysine and 1tyrosine, is a drug used for treating multiple sclerosis [12]. Schwartz's group demonstrated that GA could be used to promote an immunemediated neuroprotective response in different models of acute CNS injuries and neurodegenerative diseases $[13,14]$. A brief treatment with GA induced a weak Th1 response that may decrease neurodegeneration, whereas a prolonged treatment resulted in a Th2 response that may decrease autoimmune disease [15]. In a recent paper, Baruch et al. [16] investigated the effect of daily- versus weekly-GA administration for 4 weeks in a murine model of Alzheimer disease. Their results indicated that the weekly scheme resulted in a beneficial effect on both cognitive performance and plaque clearance. In contrast, daily-treatment did not show any positive effect, even more they found a clear tendency to an adverse effect.

We previously demonstrated that weekly treatment with GA was able to reverse the deleterious effects of chronic stress restoring the physiological levels of adult neurogenesis and Th1/Th2 peripheral balance [3]. In addition, recent reports have showed that activation of immune system by Influenza A (H1N1) vaccination during early pregnancy $[17,18]$, or by neonatal BCG vaccination [19] enhanced neurogenesis in early life in mice by regulation of the neuroimmune milieu in the brain related to a systemic Th1 bias.

The present study was performed to investigate if peripheral immune changes correspond to altered cytokine and neurotrophin expression in the hippocampus that in turn influence the memory performance. Furthermore, we analyzed if the molecular changes in hippocampus are also found in lymphocytes in order to propose these cells as peripheral markers of cognitive deficit and of response to treatment. It is important to consider that Th1 and Th2 cytokines are mainly synthetized by $\mathrm{T}$ lymphocytes and that lymph nodes have a higher percentage of T-lymphocytes than spleen. Moreover, lymph nodes have a similar percentage of $\mathrm{T}$ cells than peripheral blood. Taking into account these facts lymph nodes were used as the peripheral structure to perform this study.

\section{Material and methods}

\subsection{Animals}

Two-months-old female BALB/c mice were acquired from the Veterinary School of the University of Buenos Aires (Argentina) and maintained in the animal facility of School of Medicine of University of Buenos Aires (Argentina). The mice were maintained with lights on at 07:00 and off at 19:00 under controlled temperatures $\left(20 \pm 2{ }^{\circ} \mathrm{C}\right)$. Food and water were available ad libitum except when it is indicated. Animals were taken care of and sacrificed according to the rules of the "Guide for the Care and Use of Laboratory Animals" (NIH) (revision 2011) and to the EC Directive 86/609/EEC (revision 2010). The experimental protocol was also approved by the Institutional Committee for the Use and Care of Laboratory Animal rules (CICUAL, School of Medicine, University of Buenos Aires, Argentina) under resolution $2947 / 13$

\subsection{Chronic mild stress model}

The stress protocol was slightly modified from Monleon et al. [20]. Each mouse was housed individually and exposed to different stressors: one period of water $(16 \mathrm{~h})$ and food $(8 \mathrm{~h})$ deprivation, solid cage $(17 \mathrm{~h})$, grouping $(17 \mathrm{~h})$, and two period of $45^{\circ}$ cage tilting ( 7 and $17 \mathrm{~h}$ ), continuous overnight illumination. All individual stressors used were classified as "mild" according to the Animals Scientific Procedures Act of 1986 (UK legislation). The individual stressors were dispersed throughout 9 weeks in a similar manner to that previously described [9].

\subsection{GA treatment}

GA consists of acetate salts of synthetic polypeptides containing 1alanine, l-glutamate, 1-lysine and l-tyrosine [12]. After 6 weeks of stress exposure, the animals were injected with GA for 3 weeks. Each mouse received four subcutaneous injections of $100 \mu \mathrm{g}$ of GA dissolved in phosphate-buffered saline (PBS) at a final volume of $150 \mu$, according to the procedure described by Butovsky et al. [21]. During the first week of the GA treatment, the animals were injected twice at a 24-h interval and once per week for the following 2 weeks. GA (Escadra ${ }^{\circledR}$ 325032A batch no. 70253, MR PHARMA S.A., Malvinas Argentinas, Buenos Aires, Argentina) was kindly provided by Raffo, Argentina. As indicated below, the stress exposure was continued after the GA injection to avoid any effect due to the lack of stress [3]. PBS was used as the control vehicle. The mice were tested 7 days after the last GA injection.

\subsection{Experimental design}

The mice were randomly divided into two groups: one that was housed in normal conditions (control mice, $\mathrm{n}=35$ ) and another that was exposed to the chronic mild stress model (CMS mice, $n=35$ ). Six weeks later, the control and CMS mice were injected with PBS or GA. Four experimental groups were formed: control PBS $(n=17)$, control GA $(n=18)$, CMS PBS $(n=18)$ and CMS GA $(n=17)$. The chronic stress protocol (see above) was continued upon the GA treatment to rule out any effect due to the lack of stress. Mice of each group were used to evaluate behavioral performance and cytokine and neurotrophin mRNA levels in both hippocampus and lymphoid nodes. To avoid any influence of Barnes maze procedure on cytokine and neurotrophin levels, different animals were used in Barnes maze and qRT-PCR analysis. The experimental protocols were approved by the Institutional Committee for the Use and Care of Laboratory Animal (CICUAL, School of Medicine, University of Buenos Aires, Argentina).

\subsection{Barnes maze}

The performance in the Barnes maze was evaluated in the four experimental groups: control PBS $(n=7)$, control GA $(n=8)$, CMS PBS $(n=8)$ and CMS GA $(n=7)$. The Barnes maze assesses spatial learning and memory and it is based on the natural tendency of mice to explore and escape through holes [22]. The paradigm performed here was carried out in stages over 7 days, and included habituation (on day 1), acquisition (days 2-6) and probe testing (day 7). The Barnes maze consisted on a circular, grey-coated opaque platform $90 \mathrm{~cm}$ in diameter and elevated $75 \mathrm{~cm}$ above the floor. Sixteen $5 \mathrm{~cm}$ wide holes were evenly distributed around the perimeter, $5 \mathrm{~cm}$ from the edge. The scape box $(17 \mathrm{~cm} \times 13 \mathrm{~cm} \times 7 \mathrm{~cm})$ was a grey store box with a $5 \mathrm{~cm}$ diameter hole in the lid and represented the target [23]. Distinct spatial cues are located all around the maze and kept constant throughout the study. Habituation and acquisition periods consisted of four trials/day for six consecutive days with 20 min inter-trial interval. The mouse was placed in the middle of the maze in a $10 \mathrm{~cm}$ high cylindrical black start chamber. After $10 \mathrm{~s}$, the start chamber was removed, three $60 \mathrm{~W}$ bulbs were turned on and the mouse was set free to explore the maze. The trial ended when the mouse entered the escape box or after $5 \mathrm{~min}$ elapsed and if this failed, it was manually placed inside for $15 \mathrm{~s}$. When the mouse entered the escape box, the light was turned off, and it was quickly carried to the home cage. The escape box was always located underneath the same hole (stable within the spatial environment), which was randomly determined for each mouse. In between each trial, the maze was cleaned with $70 \%$ ethanol to remove odor cues. Day 1 of habituation was not included in the analysis of the data due to the great dispersion that the variables presented during this day. The measures recorded in acquisition trials included the amount of time that the mice spends searching in each quadrant, the escape latency to enter the box 
with all four paws and number of errors made before entering the escape hole. The quadrants were defined as: target (quadrant that contain the escape hole with the scape box), right, opposite and left (right, opposite and left quadrant respect to target quadrant respectively). Errors were defined as nose pokes and head deflections into a hole not leading to the scape box. Reference memory errors were recorded when a mouse made a nose and head deflection into a non-escape hole, whereas working memory errors were recorded when a mouse made a nose and head deflection into a non-escape hole already visited during that same trial. In addition, the type of search strategy employed by mice on each trial was recorded as serial (searching the holes in a clockwise or counterclockwise order), random (hole searches separated by crossing through the center of the maze or unorganized search), or direct search (moving directly to the escape hole or within the two adjacent holes before visiting the escape hole). On day 7 (probe testing), the escape box was removed, and the mouse was allowed to freely explore the maze for $3 \mathrm{~min}$. The recorded variables were the time spent in each quadrant, the latency to search the hole that contained the escape box in the acquisition phase and the number of errors. Then the percentage of time spent in the target quadrant (i.e., the one that originally contained the escape box) was calculated and compared with the average percent time in the other three quadrants [24,25]. A digital video camera placed above the maze recorded the animals' behavior. Data were obtained by manual observation of the videos.

\subsection{Quantitative real-time PCR ( $q R T-P C R$ )}

\subsubsection{RNA isolation}

Lymph nodes (axillary, inguinal and mesenteric) and brain were removed from control PBS, control GA, CMS PBS and CMS GA $(n=10$, each group) sacrificed mice, 7 days after the last GA injection. Hippocampi were dissected on ice according Mouse Brain Anatomy Atlas. Tissues were stored at $-80^{\circ} \mathrm{C}$ until use. Total RNA was isolated from hippocampus and lymph nodes by homogenization with $0.5 \mathrm{ml}$ of Trizol Reagent (Invitrogen, Life technologies, California, USA) following manufacturer's instructions. The total RNA was quantified by spectrometry. The relation $260 / 280 \mathrm{~nm}$ was verified for each sample.

\subsubsection{Reverse Transcription-Polymerase Chain reaction (RT-PCR)}

The total RNA $(3.5 \mu \mathrm{g})$ was used as a template to generate firststrand cDNA synthesis using the M-MLV Reverse Transcriptase (Invitrogen, Life technologies, California, USA), random primers (Invitrogen, Life technologies, California, USA) and dNTPs (Invitrogen, Life technologies, California, USA). Total RNA was reverse transcribed in a final volume of $20 \mu \mathrm{l}$ at $42^{\circ} \mathrm{C}$ for $60 \mathrm{~min}$ and $90^{\circ} \mathrm{C}$ for $10 \mathrm{~min}$ in a thermal cycler (Veriti, Applied Biosystems, Thermo Fisher Scientific, California, USA).

\subsection{3. $q R T-P C R$}

Real-time qPCR was performed using KAPA SYBR ${ }^{\circledast}$ FAST qPCR Kit Master Mix $(2 \times)$ Universal (Kapa Biosystems, Massachusetts, USA) according to manufacturer's instructions. The sequences of mousespecific primers (Integrated DNA Technologies), the accession number, the amplicon size, the annealing temperature and the melting temperature are listed in Table 1. All experiments were performed in duplicate. The PCR reaction mixture was run in the 7500 Real-Time PCR System (Applied Biosystems, Massachusetts, USA). The thermal cycling conditions included an initial denaturizing step at $95^{\circ} \mathrm{C}$ for $5 \mathrm{~min} ; 40$ cycles of $15 \mathrm{~s}$ at $95^{\circ} \mathrm{C}$ for denaturation, $20 \mathrm{~min}$ at temperature indicated in Table 1 for annealing, and $30 \mathrm{~s}$ at $72^{\circ} \mathrm{C}$ for extension, followed by melting curves to differentiate non-specific primer-dimmers and specific amplicon. The mRNA was quantified using the comparative cycle threshold (Ct) method [26]. Quantitative PCR data for each sample were normalized according to glyceraldehyde 3-phosphate dehydrogenase (GAPDH, reference gene) from the same sample. Relative changes of mRNA amount were calculated using the $2^{(-\Delta \Delta \mathrm{Ct})}$ method.
Each cDNA sample was tested for target genes of interest and the GAPDH. To verify that the reagents were not contaminated with DNA, or that the primers do not produce a signal by dimmer formation, a notemplate control sample was included for each primer set. Amplicons were analyzed by $2 \%$ agarose gel electrophoresis.

\subsection{Statistical analysis}

Data are presented as the mean \pm standard error of the mean (SEM) for each group. All the data were processed using STATISTICA software (StatSoft, Inc., Tulsa, Oklahoma, USA). The normality and homogeneity of variance for the dataset were tested using the ShapiroWilk test and Levene's test, respectively. Data were analyzed using General Linear Model (GLM) when three or more factors were included or two-way ANOVA to examine the significance of the main effects and their interactions, as appropriate.

Barnes maze data were analyzed with the GLM repeated measures (RM) analysis. The factors used according to the analyzed variable are described below. To analyze the percentage of time spent in each quadrant in the Barnes maze in the acquisition phase, the following factors were used: quadrant (target, right, opposite or left), time (day 2, $3,4,5$ or 6 ), condition (control or CMS) and treatment (PBS or GA). The latency to escape and the mean of the number of reference were analyzed used time (day 2, 3, 4, 5 or 6), condition (control or CMS) and treatment (PBS or GA) as factors. Working errors data were not normally distributed, so a non-parametric statistic Kruskal-Wallis test was performed. To analyze the percentage of search strategy, strategy (direct, random or serial), time (day 2, 3, 4, 5 or 6), condition (control or CMS) and treatment (PBS or GA) were used as factors.

To evaluate the performance in testing phase (7 day) in the Barnes maze and qRT-PCR data were analyzed with two-way ANOVA using the factors condition (control or CMS) and treatment (PBS or GA). In all cases, if ANOVA showed significant differences between groups, Fisher's Least Significant Difference (LSD) post-hoc test was performed to determine significance level. Differences between means were considered significant if $\mathrm{p}<.05$.

\section{Results}

\subsection{Chronic stress exposure alters Barnes maze performance in mice.} Reversion by GA treatment

To evaluate the effect of CMS and GA treatment on spatial learning and memory we used the Barnes maze. The Barnes maze performance was analyzed in the four groups of mice (Control PBS, Control GA, CMS PBS and CMS GA) in the acquisition (days 2-6) and test (day 7) phases.

\subsubsection{Performance in acquisition phase}

To ascertain if the treatments have an effect on the capacity to find the escape hole, we determined the percentage of total time the mice spent in each of the four quadrants, latency to escape, the number of errors and the search strategies.

Fig. 1 shows the percentage of total time the mice spent in each of the four quadrants. As it is observed, the percentage of time that mice remain in each quadrant (target, right, opposite and left quadrant) was dependent on the acquisition day (2-6). GLM repeated measure analysis indicated a significant effect of interaction day $\times$ quadrant $\left[\mathrm{F}_{(12,416)}=6.97, \mathrm{p}<.001\right]$. In addition, this parameter was dependent on mice condition (control or CMS), treatment (PBS or GA) and quadrant [interaction condition $\times$ treatment $\times$ quadrant: $F_{(3,104)}=3.51$, $\mathrm{p}<.05]$. Post-hoc test indicated control mice spent significantly more time in target quadrant respect to the others quadrants on the days 2-6 (Fig. 1a) while CMS mice increased the time that remain in the target quadrant at day 5 and 6 (Fig. 1c). These results suggest that CMS mice had a poor performance in the acquisition Barnes maze phase respect to control mice. Moreover, GA treatment improved the performance of 
Table 1

qRT-PCR primer sequence.

\begin{tabular}{|c|c|c|c|c|c|}
\hline Gene & Accession \# & Primer sequence ( $5^{\prime}$ to $\left.3^{\prime}\right)$ & Amplicon (bp) & Anneling Temp $\left({ }^{\circ} \mathrm{C}\right)$ & Melting Temp $\left({ }^{\circ} \mathrm{C}\right)$ \\
\hline IFN- $\gamma$ & NM_008337.4 & $\begin{array}{l}\text { Fw:TGCTGATGGGAGGAGATGTCTAC } \\
\text { Rv:ACCTGACACATTCGAGTGCTGT }\end{array}$ & 76 & 58 & 82 \\
\hline IL-1 $\beta$ & NM_008361.4 & $\begin{array}{l}\text { Fw: GAGGACATGAGCACCTTCTTT } \\
\text { Rv:GCCTGTAGTGCAGTTGTCTAA }\end{array}$ & 121 & 60 & 80 \\
\hline IL-2 & NM_008366.3 & $\begin{array}{l}\text { Fw:TGAGCAGGATGGAGAATTACAG } \\
\text { Rv: GAGGTCCAAGTTCATCTTCTAGG }\end{array}$ & 123 & 60 & 80 \\
\hline IL-4 & NM_021283.2 & $\begin{array}{l}\text { Fw:TTCATCGATAAGCTGCACCA } \\
\text { Rv:GCATGATGCTCTTTAGGCTTTC }\end{array}$ & 80 & 62 & 78 \\
\hline IL-6 & NM_031168.2 & $\begin{array}{l}\text { Fw:GGAAATGAGAAAAGAGTTGTG } \\
\text { Rv:GCATCCATCATTTCTTTGTAT }\end{array}$ & 109 & 60 & 77 \\
\hline IL-10 & NM_010548.2 & $\begin{array}{l}\text { Fw:GGACTTTAAGGGTTACTTGGGT } \\
\text { Rv:ATTTCTGGGCCATGCTTCT }\end{array}$ & 99 & 60 & 80 \\
\hline BDNF & NM_001285416.1 & $\begin{array}{l}\text { Fw:CTGAGCGTGTGTGACAGTATTA } \\
\text { Rv:CTTTGGATACCGGGACTTTCTC }\end{array}$ & 112 & 60 & 82.5 \\
\hline NT-3 & NM_001164035.1 & $\begin{array}{l}\text { Fw:CCTGGAAATAGTCACACGGATG } \\
\text { Rv:CTTGGATGCCACGGAGATAAG }\end{array}$ & 115 & 60 & 78.5 \\
\hline NGF & NM_001112698.2 & $\begin{array}{l}\text { Fw:CAGTGAGGTGCATAGCGTAAT } \\
\text { Rv:CTCCTTCTGGGACATTGCTATC }\end{array}$ & 107 & 60 & 80 \\
\hline GAPDH & NM_001289726.1 & $\begin{array}{l}\text { Fw:CGTCCCGTAGACAAAATGGT } \\
\text { Rv:GAATTTGCCGTGAGTGGAGT }\end{array}$ & 177 & 60 & 84.5 \\
\hline
\end{tabular}

CMS mice. As can be seen in Fig. 1d, CMS-GA mice remain more time in the target quadrant than in the others from day 3 . However, the GA treatment did not significantly affect the performance of the control mice in the acquisition phase of the Barnes maze (Fig. 1b).

As can be seen in Fig. 2, the latency to escape to the target hole is different depending on the acquisition day, condition, and treatment [Repeated measures two-way ANOVA, interaction day $\mathrm{x}$ condition $\mathrm{x}$ treatment: $\left.\mathrm{F}_{(4,104)}=3.47, \mathrm{p}<.05\right]$. Post-hoc test indicated that CMS mice lasted more time to find the escape box than control mice. Furthermore, this parameter was reverted by GA treatment in CMS mice. Control GA-treated mice did not show significant differences respect to control PBS mice.

Fig. 3 shows the reference and working errors in the acquisition phase. Repeated measures two-way ANOVA indicated that all groups of mice significantly decreased the number of reference errors through the acquisition days [Main effect of day: $\mathrm{F}_{(4,104)}=34, \mathrm{p}<.001$ ]. Moreover, a significant interaction condition $\mathrm{x}$ treatment was found $[\mathrm{F}$ $(1,26)=6.3, \mathrm{p}<.05]$. As can be seen in Fig. 3a, mice exposed to CMS showed a worse performance than control mice that is improved by GA treatment. In particular, post-hoc test indicated that CMS mice made more reference errors than control mice on day 2 and 3. Furthermore, GA treatment significantly reverted the CMS effect on day 2. Working errors are shown in Fig. 3b. A Kruskal-Wallis ANOVA indicated nosignificant differences between groups for any of the days.

We analyzed if the four experimental groups of mice used some strategy (direct, random and serial) to find the escape hole during the a.

Control PBS

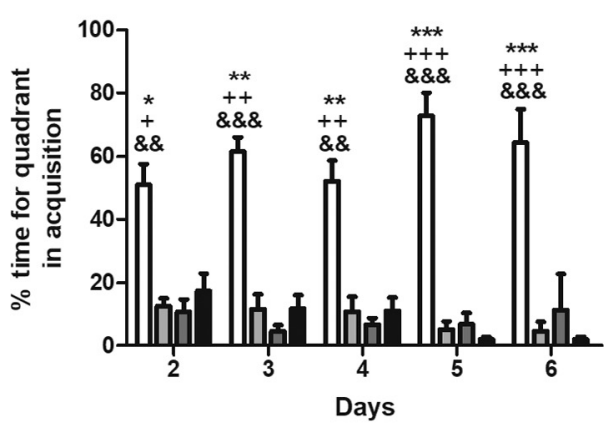

c.

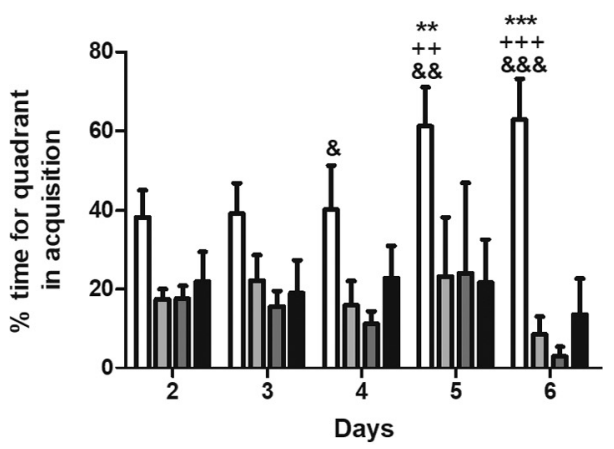

b.

Control GA

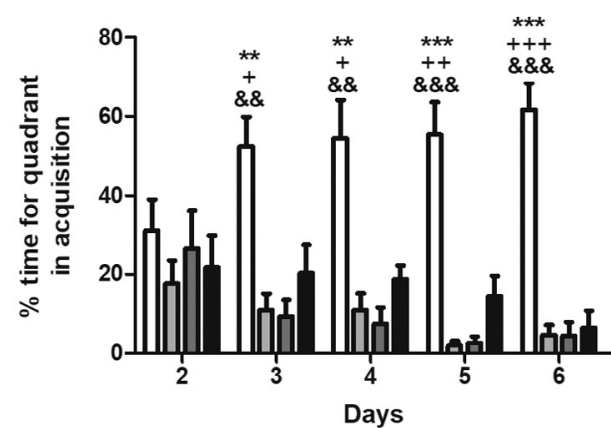

d.

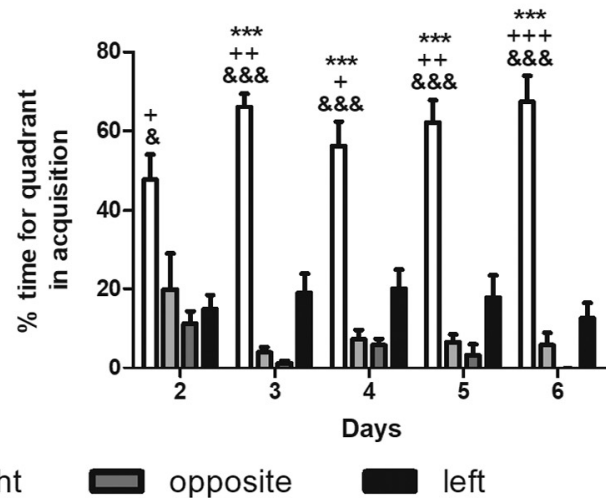

Fig. 1. Performance in acquisition phase of the Barnes maze in control, CMS and GA-treated BALB/c mice. The figure shows the percentage of time spent in the quadrants target, right, opposite and left on the days 2-6 of acquisition, measured in the four experimental groups (control PBS (a), control GA (b), CMS PBS (c) and CMS GA (d)). The bars denote the mean \pm SEM of seven or eight mice for each group. " $\mathrm{p}<.05$, ${ }^{* *} \mathrm{p}<.01,{ }^{* * * *} \mathrm{p}<.001$ respect to the right quadrant; $+\mathrm{p}<.05,++\mathrm{p}<.01+++\mathrm{p}<.001$ respect to left quadrant and $\& \mathrm{p}<.05, \quad \& \& \mathrm{p}<.01$, $\& \& \& p<.001$ compared with the opposite quadrant. 


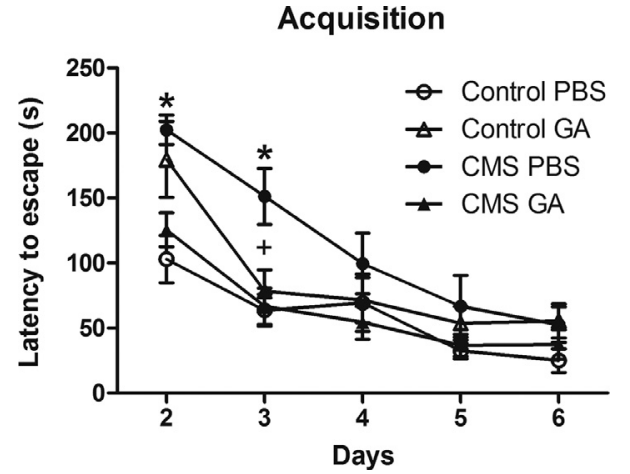

Fig. 2. Latency to escape determined during acquisition phase of the Barnes maze in control, CMS and GA-treated BALB/c mice. The graphic show the mean \pm SEM of the total latency to escape in seconds of seven or eight mice per each group. " $\mathrm{p}<.05$ respect to control PBS and $+\mathrm{p}<.05$ respect to CMS PBS.
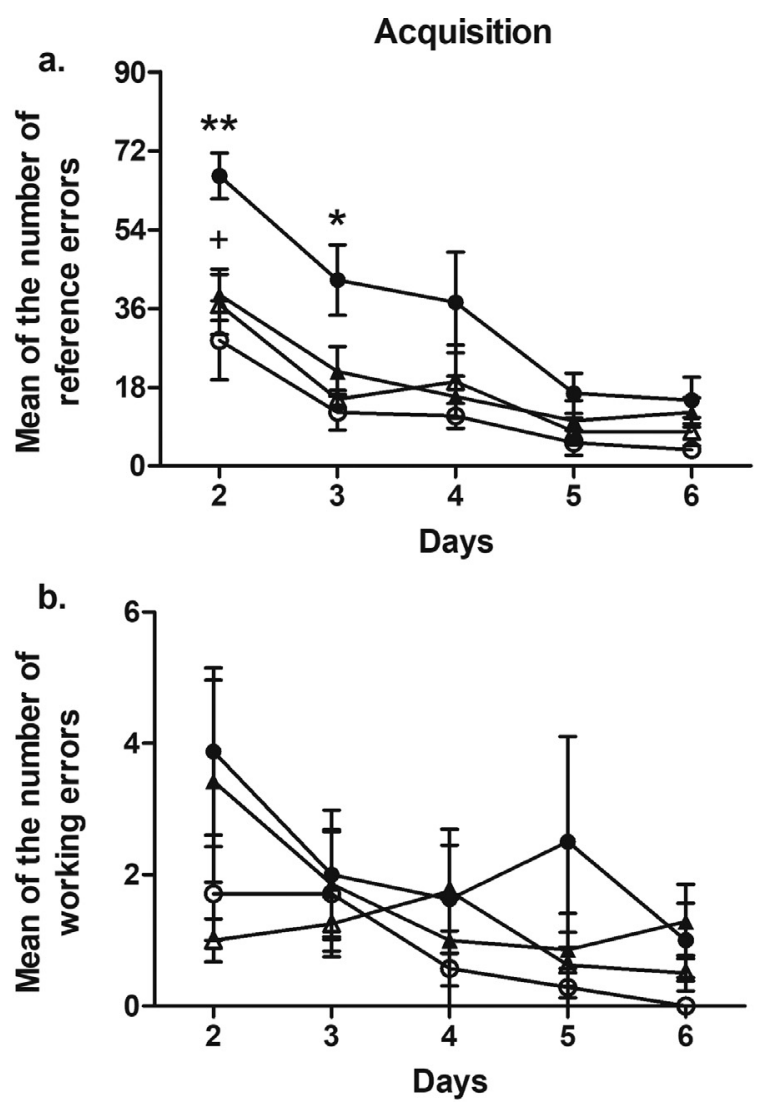

Control PBS $\triangle$ Control GA

CMS PBS

CMS GA

Fig. 3. Errors in the acquisition phase in Barnes maze in the four experimental groups. The Fig. 3 shows the reference memory errors (a) and working memory errors (b) in control and CMS BALB/c mice injected with PBS or GA. The data represent the mean \pm SEM of seven or eight mice for each group. ${ }^{*} p<.05$ and ${ }^{* *} p<.01$ with respect to the PBS-treated control mice; $+\mathrm{p}<.05$ compared with PBS-treated CMS mice.

days of acquisition in the Barnes maze (Fig. 4). GLM repeated measures analysis showed significant differences depending on the strategy (direct, random and serial), the condition, the treatment and the day [interaction day $\times$ condition $\times$ treatment $\times$ strategy: $\mathrm{F}_{(8,312)}=2.52$, $\mathrm{p}<.05]$. Post-hoc analysis showed that control PBS mice used the direct strategy in a significantly greater percentage compared with random and serial strategies on days 4,5 and 6 (Fig. 4a). On the contrary, CMS mice did not showed significant differences between the three strategies during the days $2-5$ and showed significant differences on the direct strategy respect to random strategy only on day 6
(Fig. 4c). GA treatment modified the strategy used by CMS mice. As can be seen in Fig. 4d, mice used significantly more direct strategy than the others since day 4 . Finally, GA treatment did not significantly modify the strategy used for control mice (Fig. 4b).

\subsubsection{Performance in testing phase}

On the day 7, the mice were tested without the escape box (Fig. 5). Results show that the percentage of time spent in the target quadrant was similar for all experimental groups (near 80\%, Fig. 5a). Similarly, the latency to find the target hole was not significantly different between groups [Two-way ANOVA, interaction treatment $\mathrm{x}$ condition: $\mathrm{F}_{(1,26)}=2.64$, NS; main effect, treatment: $\mathrm{F}_{(1,26)}=0.59$, NS, condition: $\mathrm{F}_{(1,26)}=0.06$, NS] (Fig. 5b). In addition, the percentage of visits to the target hole respect to total visits did not differ between the groups [Two-way ANOVA, interaction treatment $\mathrm{x}$ condition: $\mathrm{F}_{(1,26)}=2.32$, NS; main effect, treatment: $F_{(1,26)}=0.20$, NS, condition: $F_{(1,26)}=0.11$, NS] (Fig. 5c).

3.2. Alteration of cytokine but not neurotrophin profile in hippocampus and lymph nodes of chronically stressed mice

To analyze if the cognitive deficit was related to changes in cytokine and/or neurotrophin levels in hippocampus, mRNA levels of these factors were measured by qRT-PCR. Fig. 6 displays the cytokine (IFN- $\gamma$, IL-1 $\beta$ IL-2, IL-4, IL-6 and IL-10) and neurotrophin (BDNF, NT-3 and NGF) mRNA expression levels in hippocampus from mice exposed to chronic stress and treated or not with GA. For IFN- $\gamma$, two-way ANOVA indicated a different response depending on the condition and the treatment [interaction: $\mathrm{F}_{(1,36)}=12.97, \mathrm{P}<.001$ ]. Post-hoc analysis showed a significant decrease for CMS mice respect to control that was reverted by GA treatment. Similarly, IL-1 $\beta$ was modified depending on the condition $\left[\mathrm{F}_{(1,36)}=9.02, \quad \mathrm{P}<.01\right]$ and the treatment $\left[\mathrm{F}_{(1,36)}=5.44, \mathrm{P}<.05\right]$. Post-hoc test indicated a significant diminution for CMS mice respect to control and a GA-mediated return to control values. IL-4 mRNA values depended on treatment $\left[\mathrm{F}_{(1,36)}=5.75, \mathrm{P}<.05\right]$ and post-hoc test showed a non-significant effect for CMS mice respect to control but a significant increase for CMS GA mice reaching control mice values. Non-significant effects were found for IL-2, IL-6 or IL-10 mRNA levels [main effects, IL-2, condition: $\mathrm{F}_{(1,36)}=1.28$, NS, treatment: $\mathrm{F}_{(1,36)}=1.46$, NS; IL-6: $\mathrm{F}_{(1,36)}=0.16$, NS and $F_{(1,36)}=1.02$, NS; IL-10: $F_{(1,36)}=0.16$, NS and $F_{(1,36)}=0.0002$, NS]. Finally, BDNF, NT-3 and NGF mRNA levels were not significantly modified neither for the condition $\left[\mathrm{F}_{(1,28)}=0.23\right.$, NS; $\mathrm{F}_{(1,28)}=0.19$, NS; and $\mathrm{F}_{(1,28)}=0.52$, NS; respectively] nor for the treatment $\left[\mathrm{F}_{(1,28)}=0.55\right.$, NS; $\mathrm{F}_{(1,28)}=0.34, \mathrm{NS} ; \mathrm{F}_{(1,28)}=2.90$, NS; respectively $]$.

Furthermore, we analyzed if the molecular changes in hippocampus are also found in lymph nodes. Fig. 7 shows the results for the cytokine and neurotrophin mRNA expression in lymph nodes from all groups of mice. For IFN- $\gamma$, a different response was observed depending on the condition and the treatment [Two-way ANOVA, interaction: $\left.\mathrm{F}_{(1,36)}=8.59, \mathrm{P}<.01\right]$. Post-hoc analysis showed a significant decrease for CMS mice respect to control and a GA-mediated reversion was also observed. IL-4 was modified depending on the condition $\left[\mathrm{F}_{(1,36)}=4.11, \quad \mathrm{P}<.05\right]$ and the treatment $\left[\mathrm{F}_{(1,36)}=14.57\right.$, $\mathrm{P}<.001]$. Post-hoc test indicated a significant increase for CMS mice respect to control and a return to control values by GA treatment. Nonsignificant effects were found for the others cytokines [main effects, IL$1 \beta$, condition: $F_{(1,36)}=1.40$, NS, treatment: $F_{(1,36)}=0.09$, NS; IL-2: $\mathrm{F}_{(1,36)}=1.15$, NS and $\mathrm{F}_{(1,36)}=0.65$, NS; IL-6: $\mathrm{F}_{(1,36)}=0.04$, NS and $\mathrm{F}_{(1,36)}=0.23$, NS; IL-10: $\mathrm{F}_{(1,36)}=0.51$, NS and $\mathrm{F}_{(1,36)}=0.82$, NS]. In addition, BDNF, NT-3 and NGF mRNA levels were not significantly modified neither for the condition $\left[\mathrm{F}_{(1,28)}=1.21\right.$, NS; $\mathrm{F}_{(1,28)}=0.37$, NS; and $\mathrm{F}_{(1,28)}=0.0001$, NS; respectively] nor for the treatment $\left[\mathrm{F}_{(1,28)}=0.06, \mathrm{NS} ; \mathrm{F}_{(1,28)}=1.14, \mathrm{NS} ; \mathrm{F}_{(1,28)}=1.24\right.$, NS; respectively $]$. 


\section{a. Search strategies control PBS mice acquisition}

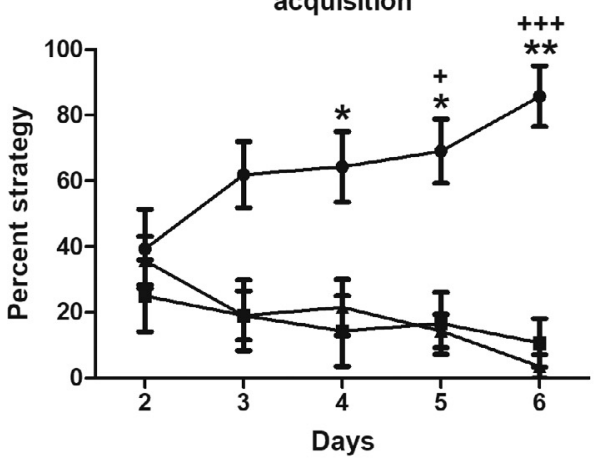

C. Search strategies CMS PBS mice acquisition

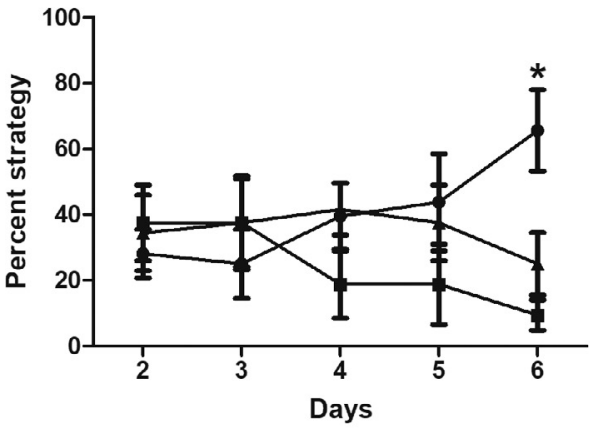

$\multimap$ Direct b. Search strategies control GA mice acquisition

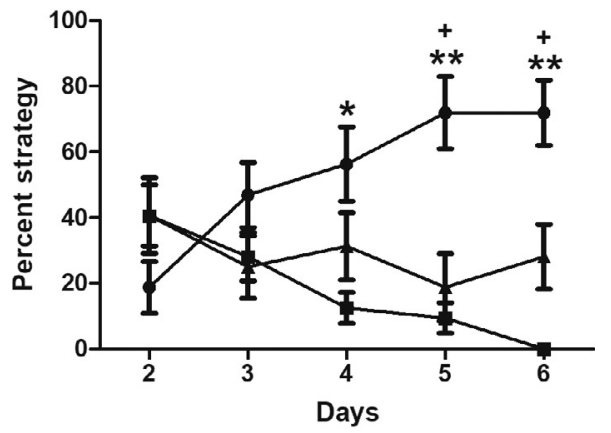

d. Search strategies CMS GA mice acquisition

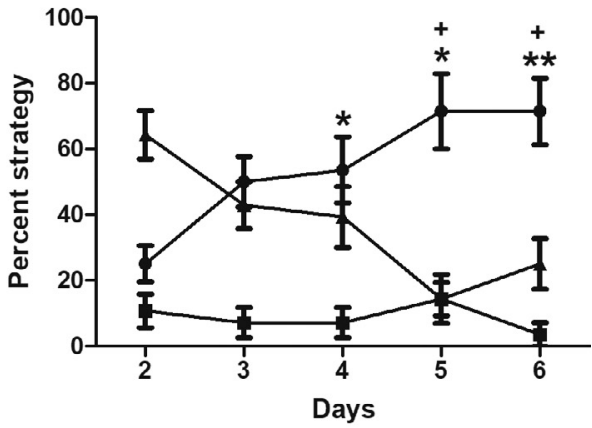

Random $₫$ Serial
Fig. 4. Search strategies in the Barnes maze in control and CMS mice and PBS and GA treatment. The graphics represent the percent of the strategy (direct, random and serial) used to find the escape hole in each day during the acquisition phase in the four experimental groups (control PBS (a), control GA (b), CMS PBS (c) and CMS GA (d) mice). The data represent the mean \pm SEM of seven or eight mice for each group. p $<.05$ and ${ }^{* *} \mathrm{p}<.01$ direct vs random; $+\mathrm{p}<.05$ and $+++\mathrm{p}<.001$ direct vs serial.

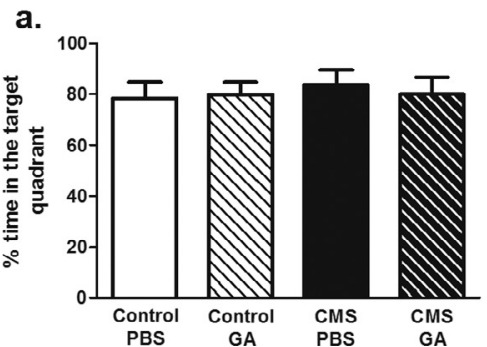

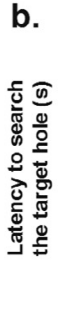

\section{Testing phase}

b.

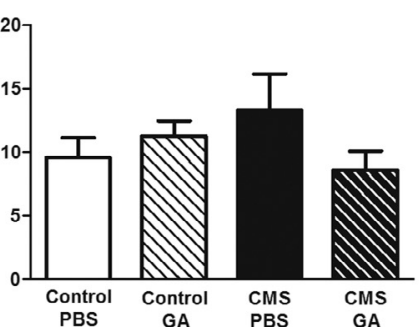

c.

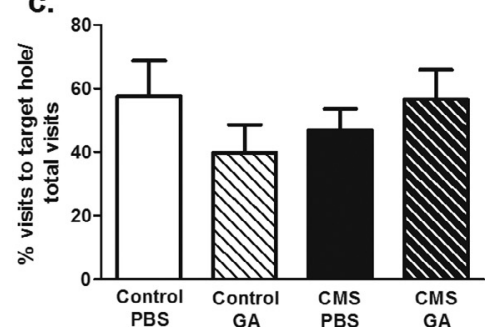

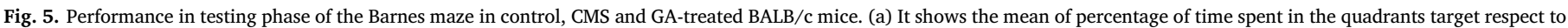

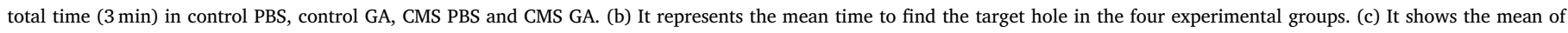
percentage of visits to the target hole respect to total visit in the testing phase. The bars denote the mean \pm SEM of seven or eight mice for each group.

\section{Discussion}

This work shows that exposure to chronic mild stress (CMS) induced a poor performance in Barnes maze task. Moreover, CMS modified the mRNA levels of cytokines in both hippocampus and lymph nodes. In addition, GA-treatment improved Barnes maze performance which was matching with the return of mRNA cytokines levels to control values in both tissues. These results suggest that cytokines, in particular IFN- $\gamma$, could be used as peripheral markers of cognitive deficit and its treatment.

In a previous report, we found that CMS mice displayed poor learning in short-memory tasks, such as open-field, passive avoidance, spontaneous alternation behavior and object-in-place task [9-11]. In agreement, in the present work, we found that chronic mild stress induced poor performance compared with control mice in Barnes maze. Stressed mice, had a greater latency to escape and more errors in their search during the acquisition trials. However, it is important to note that both control and CMS mice, learned to locate the target hole, taking into account that no significant differences were found in the parameters registered at testing day. Nevertheless, CMS mice needed more time of training to acquire the ability to find the target hole. Additionally, stressed mice did not show preference for a particular search strategy during the acquisition phase compared with control mice, which showed a direct search strategy during most acquisition days. Taken together, these results demonstrate that CMS mice had impairment in spatial learning and memory.

Cytokines and neurotrophins are thought to be important mediators of the effects of peripheral immune activation on brain development and function. Yang et al. [19] results indicated that peripheral immune activation by neonatal BCG vaccination increases neurogenesis and improves behavior through the changed expression of cytokines and neurotrophins in the hippocampus in mice. In addition, it was shown that influenza vaccines administered during early pregnancy in female mice increased hippocampal neurogenesis and neurotrophin levels in offspring $[17,18]$. It was proposed that periphery-derived cytokines and/or immune cells may penetrate into the brain and directly affect neuronal activity [27]. Moreover, the cytokines in the brain may have crosstalk with the resident immune cells (e.g., microglia) and regulate 


\section{Hippocampus}

a.

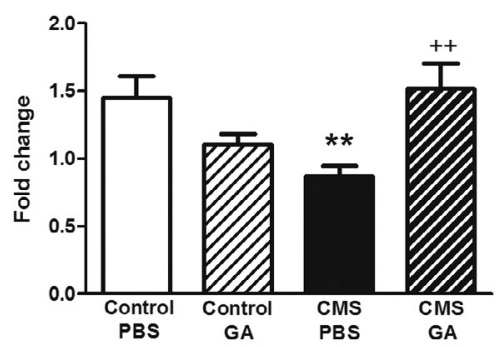

d.

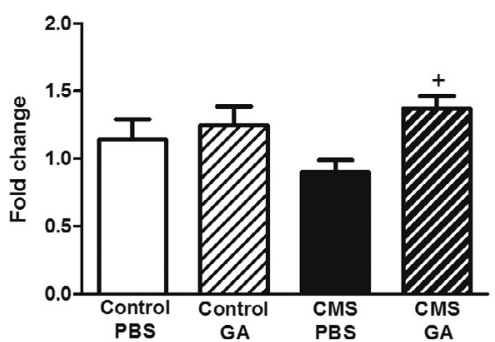

g.

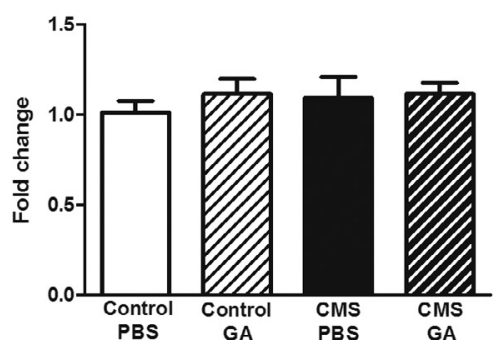

b.

IL-1 $\beta$

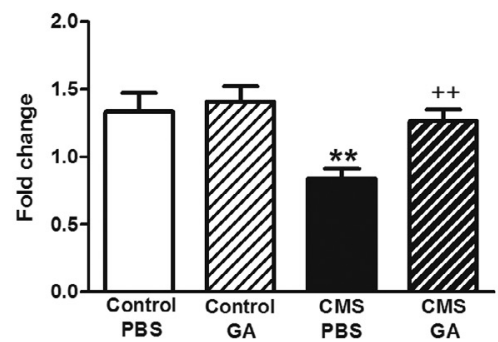

e.

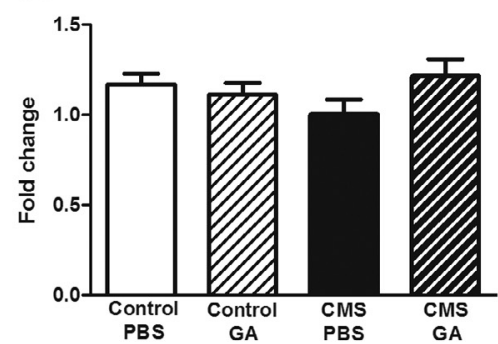

h.

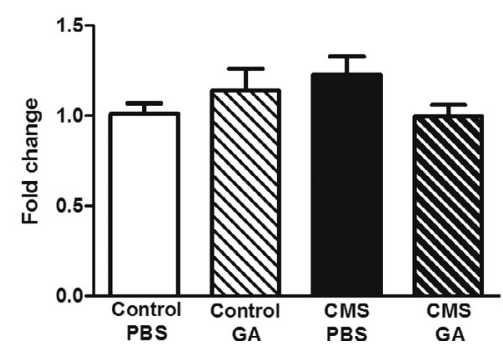

c.

IL-2

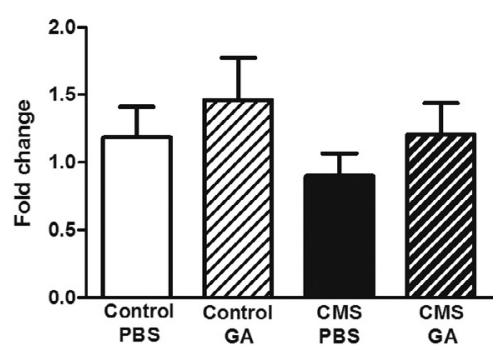

f.

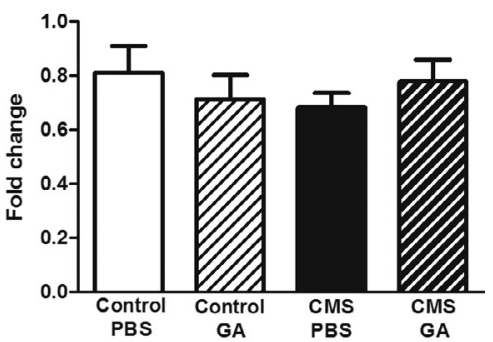

i.

NGF

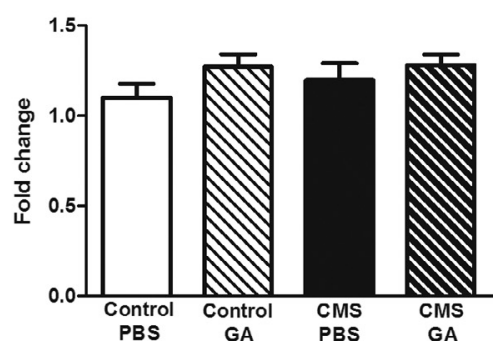

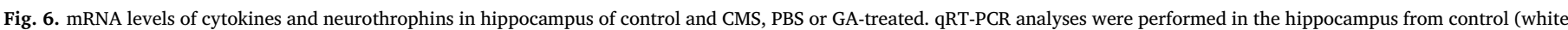

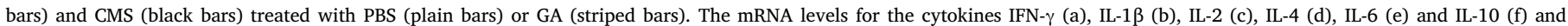

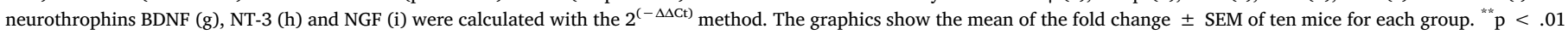
with respect to control PBS; $+\mathrm{p}<.05$ and $++\mathrm{p}<.01$ compared with CMS PBS.

their phenotypes by altering their secretion of local neuromodulatory molecules, including cytokines and neurotrophins [28]. Thus, Butovsky et al. [21] showed that the microglia activated by IL-4 can induce a bias towards oligodendrogenesis, whereas IFN- $\gamma$ activated microglia promotes neuronal differentiation. In this context, it was reported that cognitive deficits were related to a systemic Th1/Th2 production imbalance and could be reversed when balance is restored $[3,29,30]$.

In accordance to our previous results, a decrease of IFN- $\gamma$ and increase of IL-4 in mRNA levels was found in lymph nodes. However, in hippocampus, a decrease of IFN- $\gamma$ and IL- $1 \beta$ levels was found. Thus, the alterations of peripheral cytokines are not exactly consistent with those found in the hippocampus. Although the mechanism remains unclear, the reasonable explanations include the differential transport of cytokines across the blood-brain barrier [27] and differential cytokine synthesis by the resident immune cells [31]. However, both peripheral and hippocampal mRNA expression alterations were reverted by weekly GA treatment. Moreover, this was in parallel with the improvement of the performance in the Barnes maze task after GA treatment.

Nowadays, significant progress in elucidating the effects of specific cytokines in the brain on learning, memory and plasticity has been made. However, there are some contradictory results concerning to the role of cytokines in modulation of memory [32]. Several studies demonstrated a critical role for IL-1 $\beta$ in the formation of hippocampal dependent memory. Thus, it was found that IL-1 $\beta$ is upregulated by a context fear conditioning [33] and long term potentiation [34]. In addition, small amounts of IL- $1 \beta$ injected centrally enhance context fear conditioning [33], passive avoidance and spatial memory [35-37]. However at pathophysiological levels, IL-1 $\beta$ can produce detrimental effects on memory [38-40]. In this scenario, Goshen et al. [33] demonstrated that IL-1 $\beta$ in hippocampal-dependent memory follows an inverted U-shaped pattern. The authors showed that slight increase of IL- $1 \beta$ in brain improves memory, while both, the excess or the decrease, results in impaired memory. Concerning to IFN- $\gamma$, it has been implicated in both neural damage and repair. High levels of IFN- $\gamma$ in the brain were found to lead to reactive gliosis, hypomyelination, and macrophage/microglial stimulation supporting the hypothesis that IFN$\gamma$ is a key mediator in immune mediated demyelinating disorders [41]. Nevertheless, it was shown that low levels of IFN- $\gamma$ in the brain could have a neuroprotective role through modulation of glial capacity to buffer excess glutamate release, promoting the protection of hippocampal neurons from excitotoxicity [42]. Moreover, Koustova et al. [43] demonstrated, in mice homozygous for a germ line deletion of the interferon-gamma gene, that the presence of IFN- $\gamma$ is necessary at some point in the inflammatory process to protect against neurodegeneration. Likewise, Baruch et al. [16], in two models of Alzheimer disease in 


\section{Lymph nodes}

a.

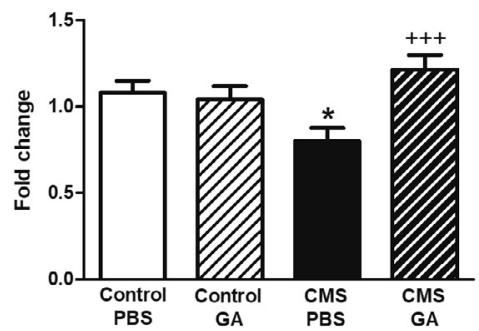

d.

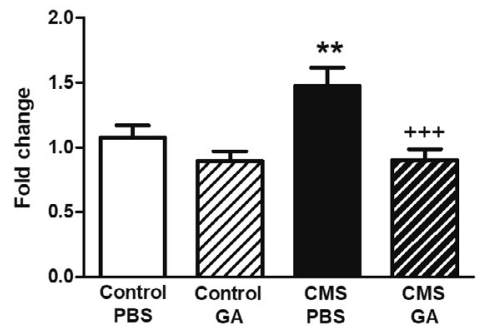

g.

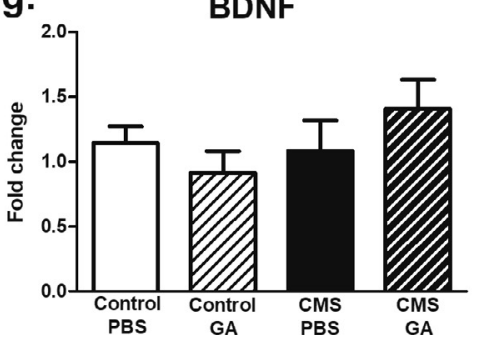

b.

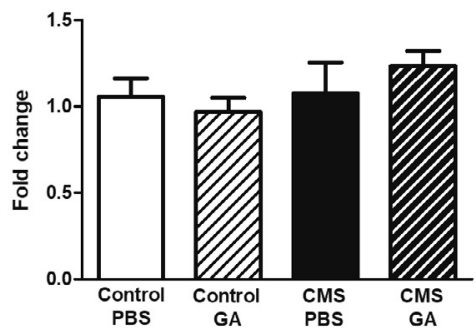

e.

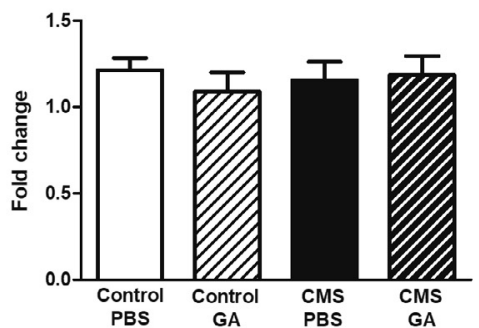

h.

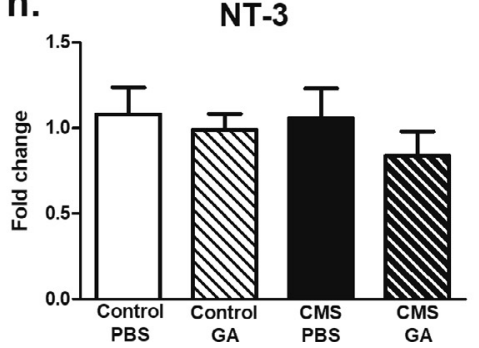

c.

IL-2

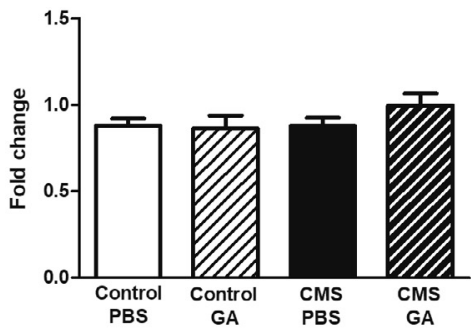

f.

IL-10

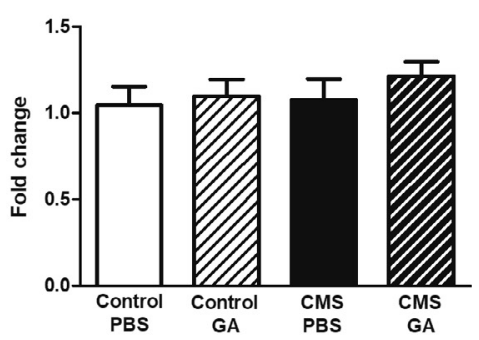

i.

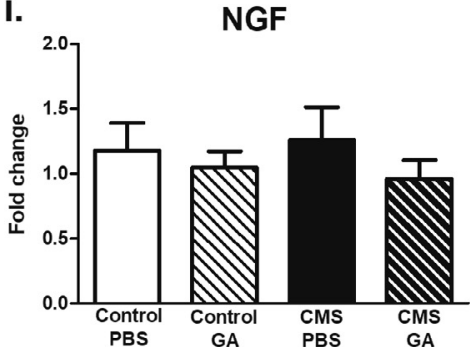

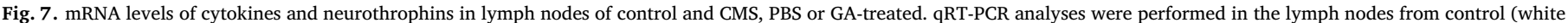

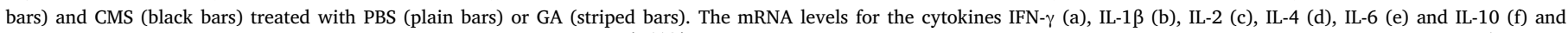

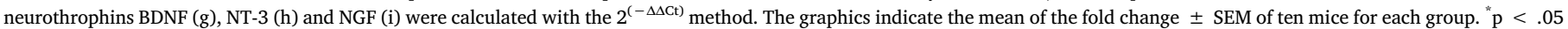
${ }^{* * *} \mathrm{p}<.01$ with respect to control PBS; $+++\mathrm{p}<.001$ compared with CMS PBS.

transgenic mice, found that during disease progression IFN- $\gamma$ levels at the brain's choroid plexus decrease, and that an experimental immune strategy to increase IFN- $\gamma$ results in disease mitigation. In addition, a recent and interesting study performed by Filiano et al. [44] suggest that IFN- $\gamma$ signaling pathway could mediate a co-evolutionary link between social/aggregation behavior and an efficient anti-pathogen response.

As it was mentioned in introduction, neurotrophins play crucial roles in the regulation of neurocognitive functions like learning and memory. However, according to our previous results [11], we did not find any alteration in neurotrophin levels neither in hippocampus nor in lymphocytes. In general, chronic stress is considered to decrease neurotrophin levels, particularly BDNF, in the hippocampus. However, the effects of stress on BDNF levels in the hippocampus appear to depend on several factors such as the type, intensity, duration and frequency of the stressors and the number of exposures [45].

Finally, our previous and present results indicate that CMS exposure induce a decreased ability to learn. In particular, in Barnes maze, mice needed more training to resolve properly the task. It is possible to postulate that CMS mice present a mild cognitive impairment. Several neurodegenerative conditions present an early mild cognitive impairment. Thus, in Alzheimer's disease (AD) it is now recognized that the $\mathrm{AD}$ pathological process begins many years before the onset of dementia and new criteria diagnostic criteria for $\mathrm{AD}$ includes the concept of mild cognitive impairment as a predementia stage of $\mathrm{AD}$ [46]. It was shown that hippocampus is affected from the earliest stages of AD [47]. Moreover, it is known that the hippocampus is critically involved in spatial memory [48]. In this scenario, it is possible to postulate that cytokines expressed in lymphoid cells could be a potential marker to detect early mild cognitive impairment. Taking into account our previous findings and results of others authors, it is possible to conclude that IFN- $\gamma$ is the main cytokine which its increase and decrease is related to improvement [16-19] and impairment [3,10,16,28,29] in cognitive performance, respectively. In this context, IFN- $\gamma$ sounds as a potential useful peripheral candidate to evaluate cognitive deficit. To give strength to this hypothesis the IFN- $\gamma$ protein levels in the serum should be tested. However we were not able to determine IFN- $\gamma$ basal levels by ELISA due to the assay sensitivity. Nevertheless, we observed in stimulated lymphocytes from peripheral blood a decrease of IFN- $\gamma$ production (near 70\%) in CMS mice that was reverted after GA treatment (unpublished data), and also we previously reported similar results in lymphoid cells from lymph node [3]. Extensive work is necessary to confirm if altered IFN-gamma levels could be used as peripheral biomarker of cognitive deficit and treatment response.

\section{Conclusion}

The present study showed that the IFN- $\gamma$ is a potential peripheral marker of cognitive deficit in the model of chronic mild stress. Moreover, these changes induced by chronic stress could be reverted by 
glatiramer acetate. Future studies are necessary to confirm and further elucidate the importance of this peripheral marker in the diagnosis and prognosis of mild cognitive deficit.

\section{Conflicts of interest}

The authors declare that they have no conflict of interest.

\section{Acknowledgements}

The authors thank Patricia Fernandez for the secretarial assistance and Daniel Gonzalez for his valuable help in the animal stress model. This work was supported by grants from CONICET - Argentina (PIP 11220110100792CO) and from the University of Buenos Aires Argentina (UBACyT 20020130100290BA) to AMG, and from the National Agency for the Promotion of Science and Technology Argentina (PICT 2013-0538) and from CONICET and the National University of the Northwest of the province of Buenos Aires - Argentina (PIO 15720150100010CO) to MLP.

\section{References}

[1] B.S. McEwen, Central effects of stress hormones in health and disease: understanding the protective and damaging effects of stress and stress mediators, Eur. J. Pharmacol. 583 (2008) 174-185.

[2] B.S. McEwen, Plasticity of the hippocampus: adaptation to chronic stress and allostatic load, Ann. NY. Acad. Sci. 933 (2001) 265-277.

[3] M.L. Palumbo, M.F. Trinchero, M.A. Zorrilla-Zubilete, A.F. Schinder, A.M. Genaro, Glatiramer acetate reverts stress-induced alterations on adult neurogenesis and behavior. Involvement of Th1/Th2 balance, Brain Behav. Immun. 26 (2012) 429-438.

[4] S. Hayley, M.O. Poulter, Z. Merali, H. Anisman, The pathogenesis of clinical depression: stressor- and cytokine-induced alterations of neuroplasticity, Neuroscience 135 (3) (2005) 659-678.

[5] Y. Dwivedi, Brain-derived neurotrophic factor in suicide pathophysiology, in: Y. Dwivedi (Ed.), The neurobiological basis of suicide. CRC Press, Boca Raton, Chapter 8. 2012; Available from: <http://www.ncbi.nlm.nih.gov/books/NBK107216/>

[6] C. Jiang, S.R. Salton, The role of neurotrophins in major depressive disorder, Trans. Neurosci. 4 (2013) 46-58.

[7] I. Kaufmann, C. Eisner, P. Richter, V. Huge, A. Beyer, A. Chouker, et al., Lymphocyte subsets and the role of TH1/TH2 balance in stressed chronic pain patients, Neuroimmunomodulation 14 (2007) 272-280.

[8] M.J. Schwarz, S. Chiang, N. Müller, M. Ackenheil, T-helper-1 and T-helper-2 responses in psychiatric disorders, Brain Behav. Immun. 15 (2001) 340-370.

[9] M.L. Palumbo, N.S. Fosser, H. Ríos, M.A. Zorrilla Zubilete, L.R. Guelman, G.A. Cremaschi, et al., Loss of hippocampal neuronal nitric oxide synthase contributes to the stress-related deficit in learning and memory, J. Neurochem. 102 (2007) 261-274.

[10] M.L. Palumbo, M.C. Canzobre, C.G. Pascuan, H. Ríos, M. Wald, A.M. Genaro, Stress induced cognitive deficit is differentially modulated in BALB/C and C57Bl/6 mice. Correlation with Th1/Th2 balance after stress exposure, J. Neuroimmunol. 218 (2010) 12-20.

[11] C.G. Pascuan, E.H. Simon, A.M. Genaro, M.L. Palumbo, Involvement of nitric oxide in improving stress-induced behavioural alteration by glatiramer acetate treatment in female BALB/c mice, Psychopharmacology (Berl). 232 (2015) 1595-1605.

[12] D. Teitelbaum, A. Meshorer, T. Hirshfeld, R. Arnon, M. Sela, Suppression of experimental allergic encephalomyelitis by a synthetic polypeptide, Eur. J. Immunol. 1 (1971) 242-248.

[13] M. Schwartz, A. London, R. Shechter, Boosting T-cell immunity as a therapeutic approach for neurodegenerative conditions: the role of innate immunity, Neuroscience 158 (2009) 1133-1142.

[14] J. Kipnis, T. Mizrahi, E. Hauben, I. Shaked, E. Shevach, M. Schwartz, Neuroprotective autoimmunity: naturally occurring $\mathrm{CD} 4{ }^{+} \mathrm{CD} 25^{+}$regulatory $\mathrm{T}$ cells suppress the ability to withstand injury to the central nervous system, Proc. Natl. Acad. Sci. USA 99 (24) (2002) 15620-15625.

[15] J. Kipnis, M. Schwartz, Dual action of glatiramer acetate (Cop-1) in the treatment of CNS autoimmune and neurodegenerative disorders, Trends Mol. Med. 8 (2002) 319-323.

[16] K. Baruch, N. Rosenzweig, A. Kertser, A. Deczkowska, A.M. Sharif, A. Spinrad, A Tsitsou-Kampeli, A. Sarel, L. Cahalon, M. Schwartz, Breaking immune tolerance by targeting Foxp3(+) regulatory T cells mitigates Alzheimer's disease pathology. Nat. Commun. 6 (2015) 7967. https://doi.org/10.1038/ncomms8967.

[17] Y. Xia, F. Qi, J. Zou, Z. Yao, Influenza A (H1N1) vaccination during early pregnancy transiently promotes hippocampal neurogenesis and working memory. Involvement of Th1/Th2 balance, Brain Res. 1592 (2014) 34-43.

[18] F. Qi, J. Yang, Y. Xia, Q. Yuan, K. Guo, J. Zou, et al., A(H1N1) vaccination recruits T lymphocytes to the choroid plexus for the promotion of hippocampal neurogenesis and working memory in pregnant mice, Brain Behav. Immun. 53 (2016) 72-83.

[19] J. Yang, F. Qi, H. Gu, J. Zou, Y. Yang, Q. Yuan, et al., Neonatal BCG vaccination of mice improves neurogenesis and behavior in early life, Brain Res. Bull. 120 (2016) $25-33$.
[20] S. Monleon, P. Aquila, A. Parra, V.M. Simon, P.F. Brain, P. Willner, Attenuation of sucrose consumption in mice by chronic mild stress and its restoration by imipramine, Psychopharmacology 117 (1995) 453-457.

[21] O. Butovsky, Y. Ziv, A. Schwartz, G. Landa, A.E. Talpalar, S. Pluchino, et al., Microglia activated by IL-4 or IFN- $\gamma$ differentially induce neurogenesis and oligodendrogenesis from adult stem/progenitor cells, Mol. Cell. Neurosci. 31 (2006) $149-160$.

[22] M.Y. Dawood, L.A. Lumley, C.L. Robison, G.A. Saviolakis, J.L. Meyerhoff, Accelerated Barnes maze test in mice for assessment of stress effects on memory, Ann. NY Acad. Sci. 1032 (2004) 304-307.

[23] K. Takao, K. Toyama, K. Nakanishi, S. Hattori, H. Takamura, M. Takeda, et al., Impaired long-term memory retention and working memory in sdy mutant mice with a deletion in Dtnbp1, a susceptibility gene for schizophrenia, Mol. Brain 1 (2008) 11.

[24] C.A. Barnes, Memory deficits associated with senescence: a neurophysiological and behavioral study in the rat, J. Comp. Physiol. Psychol. 93 (1) (1979) 74-104.

[25] K. Fabricius, G. Wörtwein, B. Pakkenberg, The impact of maternal separation on adult mouse behaviour and on the total neuron number in the mouse hippocampus, Brain Struct. Funct. 212 (5) (2008) 403-416.

[26] K.L. Livak, T.D. Schmittgen, Analysis of relative gene expression data using real time quantitative PCR and the 2KDDCT method, Methods 25 (2001) 402-408.

[27] W.A. Banks, Blood-brain barrier transport of cytokines: a mechanism for neuropathology, Curr. Pharm. Des. 11 (2005) 973-984.

[28] M. Schwartz, R. Shechter, Systemic inflammatory cells fight off neurodegenerative disease, Nat. Rev. Neurol. 6 (7) (2010) 405-410.

[29] K. Baruch, N. Ron-Harel, H. Gal, A. Deczkowska, E. Shifrut, W. Ndifon et al., CNS specific immunity at the choroid plexus shifts toward destructive Th2 inflammation in brain aging, Proc. Natl. Acad. Sci. USA. 110 (2013) 2264-2269.

[30] F. He, J.T. Zou, Q.F. Zhou, D.L. Niu, W.H. Jia, Glatiramer acetate reverses cognitive deficits from cranial-irradiated rat by inducing hippocampal neurogenesis, J. Neuroimmunol. 271 (2014) 1-7.

[31] R. Yirmiya, I. Goshen, Immune modulation of learning, memory, neuralplasticity and neurogenesis, Brain Behav. Immun. 25 (2011) 181-213.

[32] E.J. Donzis, N.C. Tronson, Modulation of learning and memory by cytokines: signaling mechanisms and long term consequences, Neurobiol. Learn Mem. 115 (2014) 68-77.

[33] I. Goshen, T. Kreisel, H. Ounallah-Saad, P. Renbaum, Y. Zalzstein, T. Ben-Hur, et al., A dual role for interleukin-1 in hippocampal-dependent memory processes, Psychoneuroendocrinology. 32 (8-10) (2007) 1106-1115.

[34] A. del Rey, D. Balschun, W. Wetzel, A. Randolf, H.O. Besedovsky, A cytokine network involving brain-borne IL-1 $\beta$, IL-1ra, IL-18, IL-6, and TNF $\alpha$ operates during long-term potentiation and learning, Brain Behav. Immun. 33 (2013) 15-23.

[35] C. Song, A.G. Phillips, B. Leonard, Interleukin 1 beta enhances conditioned fear memory in rats: possible involvement of glucocorticoids, Eur. J. Neurosci. 18 (7) (2003) 1739-1743.

[36] R. Yirmiya, G. Winocur, I. Goshen, Brain interleukin-1 is involved in spatial memory and passive avoidance conditioning, Neurobiol. Learn Mem. 78 (2) (2002) 379-389.

[37] F. Mina, C.M. Comim, D. Dominguini, O.J. Cassol, D.M. Dall'Igna, G.K. Ferreira, et al. Il1-beta involvement in cognitive impairment after sepsis. Mol. Neurobiol. 49 (2014) 1069-1076.

[38] P. Gonzalez, I. Machado, A. Vilcaes, C. Caruso, G.A. Roth, H. Schiöth, et al., Molecular mechanisms involved in interleukin 1-beta (IL-1ß)-induced memory impairment. Modulation by alpha-melanocyte-stimulating hormone ( $\alpha$-MSH), Brain Behav. Immun. 34 (2013) 141-150.

[39] A.H. Moore, M. Wu, S.S. Shaftel, K.A. Graham, M.K. O'Banion, Sustained expression of interleukin-1beta in mouse hippocampus impairs spatial memory, Neuroscience 164 (4) (2009) 1484-1495.

[40] A.M. Hein, M.R. Stasko, S.B. Matousek, J.J. Scott-McKean, S.F. Maier, J.A. Olschowka, et al., Sustained hippocampal IL-1ß overexpression impairs contextual and spatial memory in transgenic mice, Brain Behav Immun. 24 (2) (2010) 243-253.

[41] P. Maña, D. Liñares, S. Fordham, M. Staykova, D. Willenborg, Deleterious role of IFNgamma in a toxic model of central nervous system demyelination, Am. J. Pathol. 168 (5) (2006) 1464-1473.

[42] I. Shaked, D. Tchoresh, R. Gersner, G. Meiri, S. Mordechai, X. Xiao, et al., Protective autoimmunity: interferon- $\gamma$ enables microglia to remove glutamate without evoking inflammatory mediators, J. Neurochem. 92 (2005) 997-1009.

[43] E. Koustova, Y. Sei, T. McCarty, M.G. Espey, R. Ming, H.C. Morse, et al., Accelerated development of neurochemical and behavioral deficits in LP-BM5 infected mice with targeted deletions of the IFN-gamma gene, J. Neuroimmunol. 108 (2000) $112-121$.

[44] A.J. Filiano, Y. Xu, N.J. Tustison, R.L. Marsh, W. Baker, I. Smirnov, et al., Unexpected role of interferon- $\gamma$ in regulating neuronal connectivity and social behaviour, Nature 535 (7612) (2016) 425-429.

[45] F.L. Neto, G. Borges, S. Torres-Sanchez, J.A. Mico, E. Berrocoso, Neurotrophins role in depression neurobiology: a review of basic and clinical evidence, Curr. Neuropharmacol. 9 (4) (2011) 530-552.

[46] M.S. Albert, S.T. DeKosky, D. Dickson, B. Dubois, H.H. Feldman, N.C. Fox, et al., The diagnosis of mild cognitive impairment due to Alzheimer's disease: recommendations from the National Institute on Aging-Alzheimer's Association workgroups on diagnostic guidelines for Alzheimer's disease, Alzheimers Dement. 7 (2011) 270-279.

[47] J.L. Price, D.W. McKeel, V.D. Buckles, C.M. Roe, C. Xiong, M. Grundman, et al., Neuropathology of nondemented aging: presumptive evidence for preclinical Alzheimer disease, Neurobiol. Aging 30 (2009) 1026-1036.

[48] G. Iaria, J.-K. Chen, C. Guariglia, A. Ptito, M. Petrides, Retrosplenial and hippocampal brain regions in human navigation: complementary functional contributions to the formation and use of cognitive maps, Eur. J. Neurosci. 25 (2007) 890-899. 